\title{
Free-Balloon Borne Meteorological Refractometer
}

\author{
John F. Theisen and E'arl E. Gossard
}

(July 14, 1960; revised August 15, 1960)

\begin{abstract}
In this paper a free balloon borne refractometer is described and samples of soundings are shown. The instrument has been used for sampling the refractive index through the troposphere in a study of the fine structure of refractive layers over Southern California. It uses fast response tempesature and humidity elements and is carried aloft by a standard radiosonde balloon. The instrument avoids the defects of the usual radiosonde for obtaining refractive index information while allowing a nearly vertical sounding to be obtained without the disturbance of the atmosphere characteristic of a sounding taken by an aircraft.
\end{abstract}

\section{Introduction}

The vertical distribution of refractive index is of primary importance in a study of radio-wave propagation in the lower atmosphere. We have devised a system to obtain a direct and continuous measurement of refractive index by using slightly modified $400 \mathrm{Mc} / \mathrm{s}$ radiosonde components and techniques. The system was designed as a research tool to supplement information obtained from an aircraft borne microwave refractometer in an investigation of the intense super-refractive layers commonly found near the surface off the Southern California coast. There are certain well-known disadvantages to aircraft aside from expense and the necessity of maintaining air and ground crews. An aircraft being a large fast-moving vehicle disturbs the medium by an amount which is difficult to assess. Also, aircraft must necessarily pass rapidly through a very large horizontal distance in order to pass slowly through a small vertical distance. Thus fairly weak horizontal gradients may be erroneously interpreted as strong vertical gradients. These factors were important in the studies carried out at the Navy Electronics Laboratory because we were primarily interested in the possible existence of persistent microlayers of very large gradient which have sometimes been suggested to explain tropospheric radio scattered fields. For this purpose we needed essentially vertical profiles by fast response sensors providing a continuous record.

\section{Refractive Index Equation}

Because of the availability and dependability of radiosonde components a system employing wet and dry bulb sensing elements was used in otherwise unmodified radiosonde transmitters. The following approach was used in developing an expression for refractive index in terms of wet and dry bulb temperature.

1 Contribution from U.S. Navy Electronies Laboratory, San Diego, Calif.
Equation (1) relates radio refractive index to pressure, temperature, and humidity

$$
N=(n-1) \times 10^{6}=\frac{77.6 P}{T}+3.73 \times 10^{5} \frac{e}{T^{2}}
$$

$n$ is refractive index, $T$ is temperature in $\left({ }^{\circ} \mathrm{K}\right), P$ is atmospheric pressure in millibars and $e$ is vapor pressure in millibars.

If we neglect the effect of pressure variation with height and define a modified refractive index, $N_{1}$, the curve of $N_{1}$ with height will be similar to $N$ except that it will not have as great a negative slope. Its departure from $N$ can be considered to be approximately linear as in the case of $M$ and $B$, two other well-known modified refractive indices. This departure is 8.5 units per $1,000 \mathrm{ft} . \quad N_{1}$ is now a function of only temperature and humidity and is given by eq (2) where $P$ has been arbitrarily chosen as 1,000 millibars ( $\mathrm{mb})$.

$$
N_{1}=\frac{77,600}{T}+3.73 \times 10^{5} \frac{e}{T^{2}} .
$$

In examining the relationship between changes in temperature and humidity and changes in refractive index, we consider the partial derivatives of $N$ with respect to $T$ and $e$ in the differential form of (2). We have

$$
d N_{1}=\frac{\partial N_{1}}{\partial T} d T+\frac{\partial N_{1}}{\partial e} d e
$$

where

$$
d e=\frac{\partial e}{\partial T_{w}} d T_{u}+\frac{\partial e}{\partial T} d T
$$

$e$ is assumed to be given by the psychrometric formula,

$$
e=e_{w}-0.000675 P\left(T-T_{w}\right)
$$

where $e_{w}$ is the saturation vapor pressure at the 
wet-bulb temperature $T_{w}$. From Clapyron's equation

$$
\frac{\partial e_{w}}{\partial T_{w}}=\frac{5418}{T_{w}^{2}} e_{w}
$$

So finally:

$$
\begin{aligned}
d N_{1}= & -\left[\frac{7.76 \times 10^{4}}{T^{2}}+\frac{7.46 \times 10^{5} e}{T^{3}}+\frac{2.52 \times 10^{2}}{T^{2}} P\right] d T \\
& +\left[\frac{3.73 \times 10^{5}}{T^{2}}\left(\frac{5418 e_{w}}{T_{w}^{2}}+0.000675 P\right)\right] d T_{w} .
\end{aligned}
$$

Defining

$$
\begin{aligned}
& A=\frac{3.73 \times 10^{5}}{T^{2}}\left(0.782+2.00 \frac{e}{T}+0.675 \Delta P\right) \\
& B=\frac{3.73 \times 10^{5}}{T^{2}}\left(0.574+5418 \frac{e_{w}}{T_{w}^{2}}+0.675 \Delta P\right) .
\end{aligned}
$$

We can write changes in refractive index $N_{1}$ as

$$
\Delta N_{1}=-A \Delta T+B \Delta T_{w}
$$

where the coefficients $A$ and $B$ are slowly varying. $\Delta P$ represents the effect of atmospheric pressure on vapor pressure and has been chosen as the departure from $850 \mathrm{mb}$. Therefore $\Delta P=P-850$.

Although the coefficients are slowly varying, well behaved and single valued in the range of interest, they are functions of two and three variables. A complete and fully automatic analog solution of the equation would therefore require a fairly sophisticated computer. Since the instrument in its present form was designed for research purposes, it was more practical to use a simple computer and require the operator at the receivers to make occasional adjustments of the coefficients at the computer according to the ambient temperature, wet-bulb temperature and height of the balloon. This is easy in the present system since both temperature and wet-bulb temperature are available and may be recorded separately if desired. $B$ usually has to be adjusted about once every $2 \mathrm{~min}$ in the first $10,000 \mathrm{ft} \mathrm{msl}$ and $A$ about once every 5 min to ensure that errors are less than 3 percent. The accuracy can be made almost as great as desired by adjusting the coefficients more often.

For field use the computing operation should be fully automatic. For this condition it may be convenient to rewrite eq (9) as

$$
\frac{1}{A} \Delta N_{1}=-\Delta T+\frac{B}{A} \Delta T_{w}
$$

In the ratio $B / A$ the effects of pressure and temperature are much reduced and the coefficient on the right hand side of (10) can be considered to be a function of $T_{w}$ only with an error of less than 4 percent through the temperature range from 0 to $25{ }^{\circ} \mathrm{C}$. The error will actually be much less in general because of the fact that temperature and pressure usually decrease together with altitude and the overall error through the sounding is negligible for operational purposes. If a simple analog computer were designed to fit the $B / A$ function, no adjustments of coefficients would have to be made during the sounding when eq (10) is used. However, a scale factor $1 / A$ which depends weakly on temperature, wet-bulb temperature and pressure has been introduced on the left-hand side of the equation. $1 / A$ is a sensitivity factor which can range from about 0.235 to 0.280 , although the actual range over the sounding will generally be much less because temperature and pressure usually decrease together. Since the only variable coefficient is now a scale factor, the minor adjustments of the refractive index scale through the sounding can be made on the recorder record after the sounding is completed by using the known temperature, wet-bulb temperature and altitude at critical points in the sounding.

\section{Refractometer System}

A standard radiosonde system uses a thermistor to obtain temperature and a lithum-chloride strip to obtain humidity. By using a switching mechanism mechanically coupled to an aneroid cell, the meteorological sensing elements along with reference resistors are switched in and out of the measuring circuit-a grid-blocking oscillator. The oscillator frequency depends on the resistance in the circuit. Reference resistors provide altitude and calibration information.

The inaccuracy and long time constant (anywhere from 5 to 35 sec depending on temperature) of the lithium chloride strip make it unacceptable in the present application because gradients of refractive index rather than absolute values of refractive index are the important factor in most radio meteorological applications. Because of the slow response and discontinuous method of transmission of information the conventional radiosonde grossly underestimates sharp refractive index gradients and small fluctuations are usually undetectable. A system was therefore developed which has the advantages of a conventional radiosonde, namely, the expendability of the measuring units, the near vertical sounding possibilities and an ascension rate of 500 to 1,000 $\mathrm{ft} / \mathrm{min}$ but which provides a continuous record of refractive index and has a sufficiently fast response to detect microlayers and strong gradients. First considerations were given to a system using one radiosonde transmitter carrying two thermistors, one for wet-bulb and one for dry-bulb temperature. Along with appropriate circuit modifications to enter a pressure dependence term from the radiosonde baroswitch a system could be developed that transmitted refractive index directly from the single transmitter [1]. ${ }^{2}$ However, since our main interest was to have a system which required little or no

2 Figures in brackets indicate the literature references at the end of this paper. 
modification to presently existing equipment, we decided on a dual transmitter-receiver setup. This permitted use of standard transmitters and receptors. Also, previously developed test, calibration and operating procedures could be followed. In this system separate signals are telemetered to the ground by conventional radiosonde transmitters. A simple analog computer on the ground converts the temperature and humidity information to refractive index which is then recorded as a continuous function of altitude. Reference altitudes are obtained by interrupting the otherwise continuous trace at the high reference contacts of the baroswitch unit. The pressure altitude thus obtained is sufficiently accurate for radio-meteorological requirements and compares favorably with altitudes obtained from aircraft altimeters. A block diagram of the entire refractometer system is shown in figure 1 and a detailed block diagram of the computer is shown in figure 2 . Figure 3 shows the balloon-borne components of the system. Figure 4 shows the receivers, computer and recorder. The recorder was a continuously recording potentiometer type, made by Varian and the operational amplifier was a plug-in type made by Philbrick.

A wet-bulb measuring device was substituted for the lithium-chloride strip. It was a thermistor identical to the temperature element but provided with a fine cotton wick and a reservoir of distilled water. The water was fed to the element by capillary action and ventilation was provided by the vertical motion of the entire unit. A ventilation rate of $100 \mathrm{ft} / \mathrm{min}$ was required to maintain a reliable wet-bulb measurement. The time constant of this type of wet-bulb was $2 \frac{1}{2}$ sec and essentially inde-

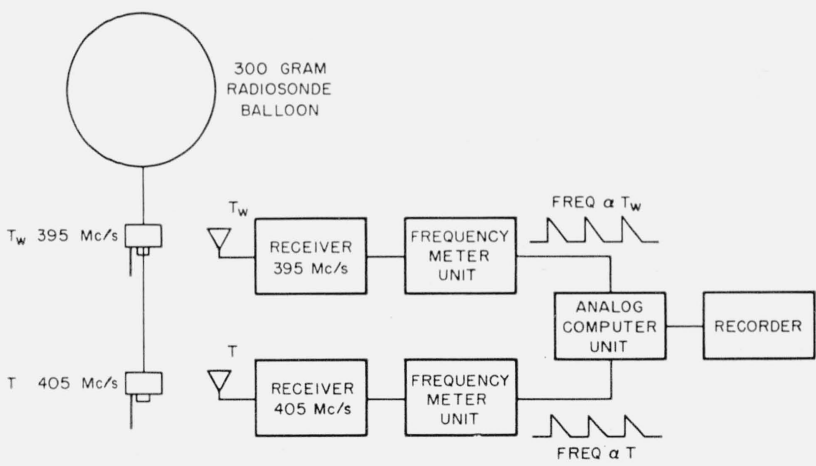

Figure 1. Block diagram of balloon-borne meteorological refractometer system.

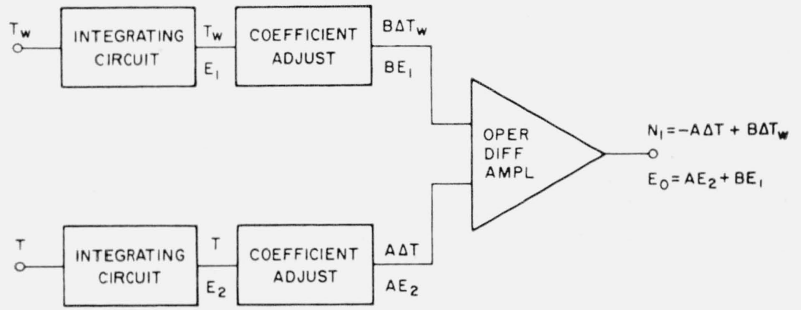

Figure 2. Block diagram of computer.

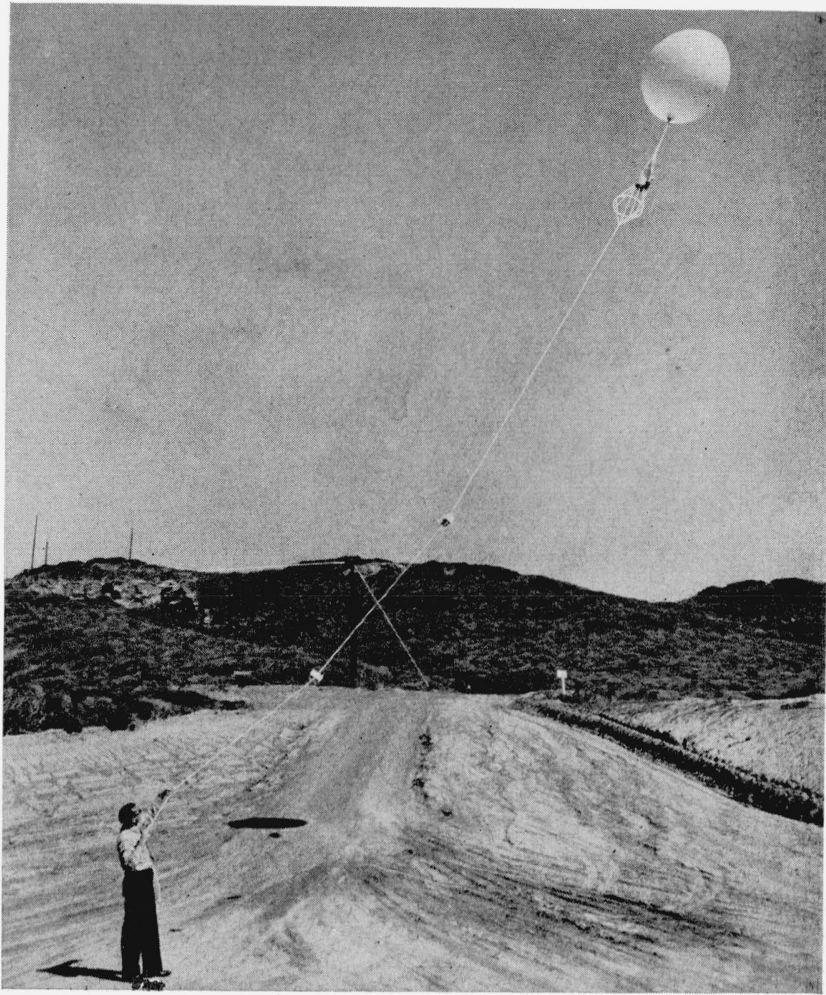

Figure 3. Photograph of the airborne components.

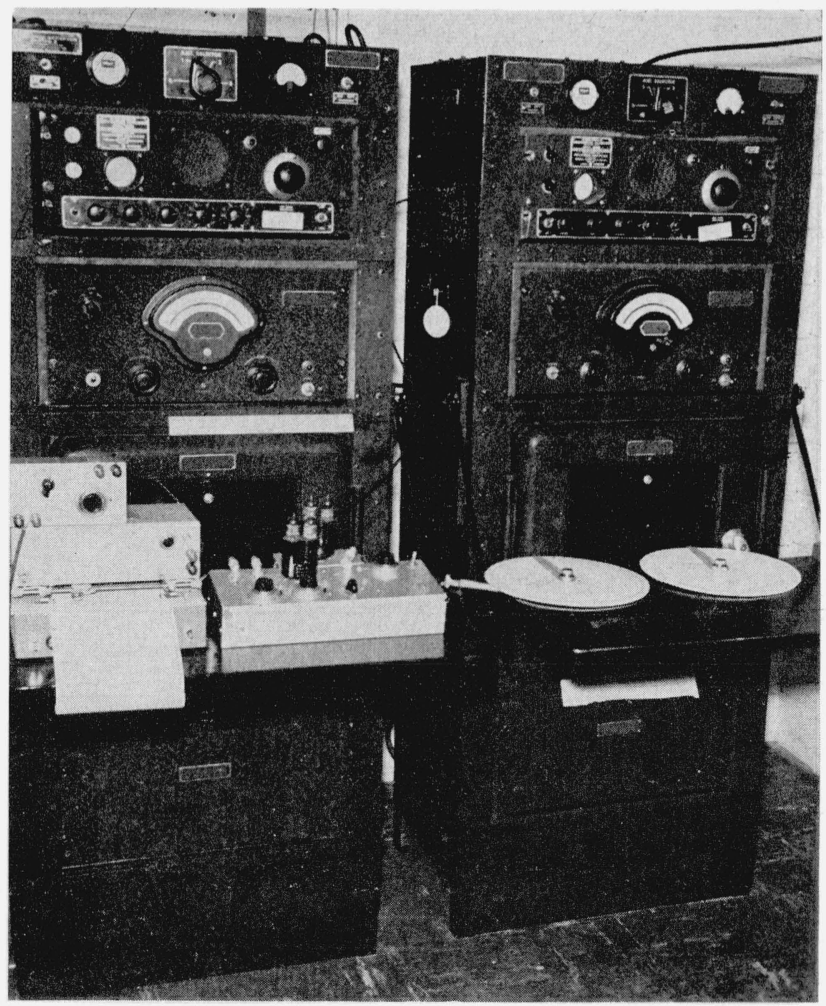

FIGURE 4. Photograph of the receivers, computer, and recorder. 
pendent of ambient temperature. The wet-bulb freezes when the air temperature is between -4 and $-8{ }^{\circ} \mathrm{C}$. The freezing is very aburpt as seen in the sample soundings shown in figure 6 . After the latent heat released has been dissipated the record quickly returns to normal. Since this system was primarily intended for use at temperatures above $0{ }^{\circ} \mathrm{C}$, no further consideration has so far been given to the characteristics of the frozen wet bulb. Over the ocean areas of the world the great majority of superrefractive layers occur in the lowest $10,000 \mathrm{ft}$ of the atmosphere [2] and the record after the wet bulb froze was of little interest in the research program for which the refractometer system was designed. In principle it should be entirely possible to make simple adjustments of the computer when the wet-bulb freezing level is reached and continue the sounding to higher elevations using the ice-bulb temperature as a measure of humidity. Tentative investigation indicates that this procedure is practical, but experimental study of the time required for the ice to sublime off of the wet bulb is needed.

Many years of use at the Navy Electronics Laboratory have proven the accuracy and reliability of carefully wrapped thermistors as wet-bulb temperature sensing elements. They have been used in captive balloon measurements $[3,4]$ and in wetthermistor rod aerographs. A refractive index variometer using a wet-bulb, dry-bulb approach

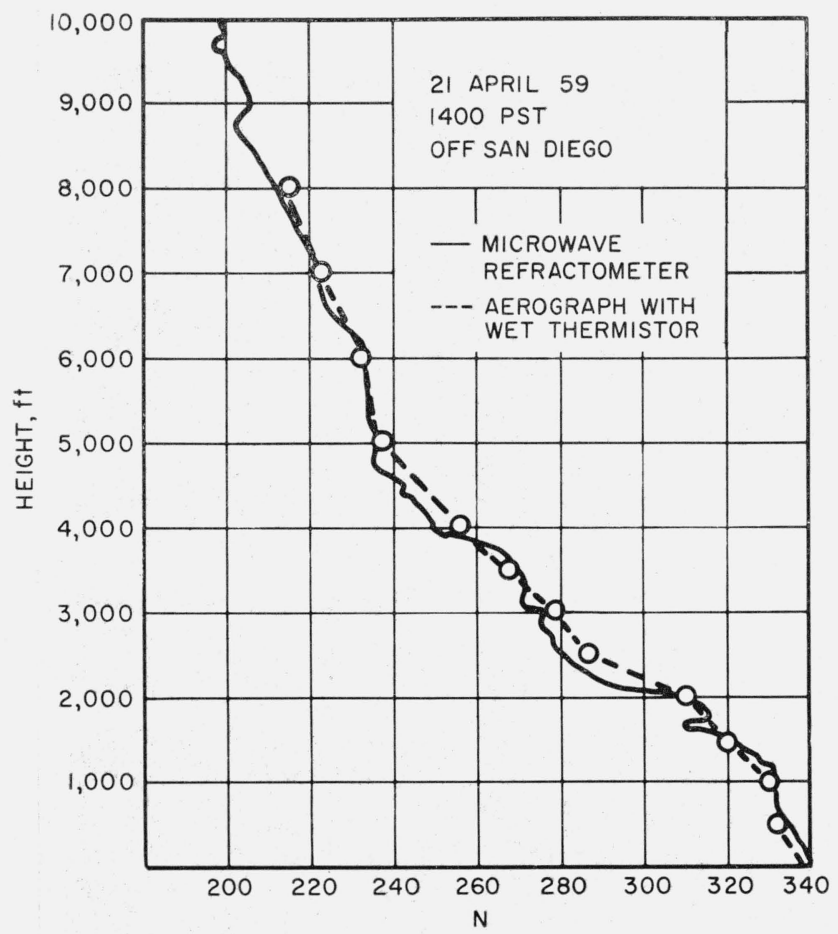

FiguRE 5. Simultaneous microwave refractometer (solid curve) and wet-thermistor aerograph soundings taken by an aircraft.

Aerograph temperature and wet-bulb temperature were read every $500 \mathrm{ft}$ and refractive index was then computed by hand. consisting of fast response thermistor beads has been developed in Japan [5] and has been used for measurements to $1,500 \mathrm{ft}$. Figure 5 shows profiles of refractive index taken simultaneously with a microwave refractometer and with an aerograph in an SNB-5 aircraft. Because of the low speed of this aircraft, frictional and compressional heating of the thermistor elements is not serious and it is seen that the agreement of the two measuring systems is excellent even though the aerograph was only read at $500-\mathrm{ft}$ intervals.

\section{Discussion of the Sounding Data}

Several of the soundings were compared with the refractive index profiles computed from radiosonde data. Figure 6 shows sample refractive index soundings. The afternoon sounding of 11 March was followed immediately by a conventional radiosonde ascent. The radiosonde record was analyzed exactly as it would normally be analyzed in a weather station and the resulting profile is shown by the straight line segment sounding in figure 7 . It is seen that several strong gradients and many small features are missed entirely by the radiosonde.

Figure 8 shows a refractive index profile in which a very abrupt change in refractive index occurs. At $600 \mathrm{ft}$ msl the refractive index changed $20 \mathrm{~N}$ units in $150 \mathrm{ft}$ or 133 units per $1,000 \mathrm{ft}$. However the local San Diego radiosonde observation indicated a change of $25 N$ units in $500 \mathrm{ft}$ or only 50 units per $1,000 \mathrm{ft}$. For many thin lavers the error in gradient has been found to be even larger.

Persistence of the atmospheric fine structure is of special interest both in tropospheric scatter propagation studies and in radar coverage prediction. The release times on 11 March (fig. 6) were $3 \mathrm{hr}$ apart. The overall similarity of the profiles is striking, and even some of the fine structure of the first sounding is repeated in the second.

One very important advantage of a balloon sounding over an aircraft sounding is its nearly vertical ascent relative to the air stream. For example it has been noticed for several years by many observers that a thin but prominent minimum in refractive index often occurs in the lower part of strong super refractive layers recorded by microwave refractometers in aircraft. It has been the generally accepted belief that this minimum was caused by the horizontal component of the aircraft's flight path as it passed through a wavy structure entering the layer. The situation is shown schematically in figure 9 . The balloon-borne system definitely establishes the fact that this interpretation is not correct because the prominent minimum often appears in the balloon soundings also. Furthermore the balloon soundings establish the fact that the small refractive index minimum is due principally to the humidity distribution. A vertical sounding by balloon thus eliminates ambiguities arising from the horizontal component of the flight path of an aircraft. 

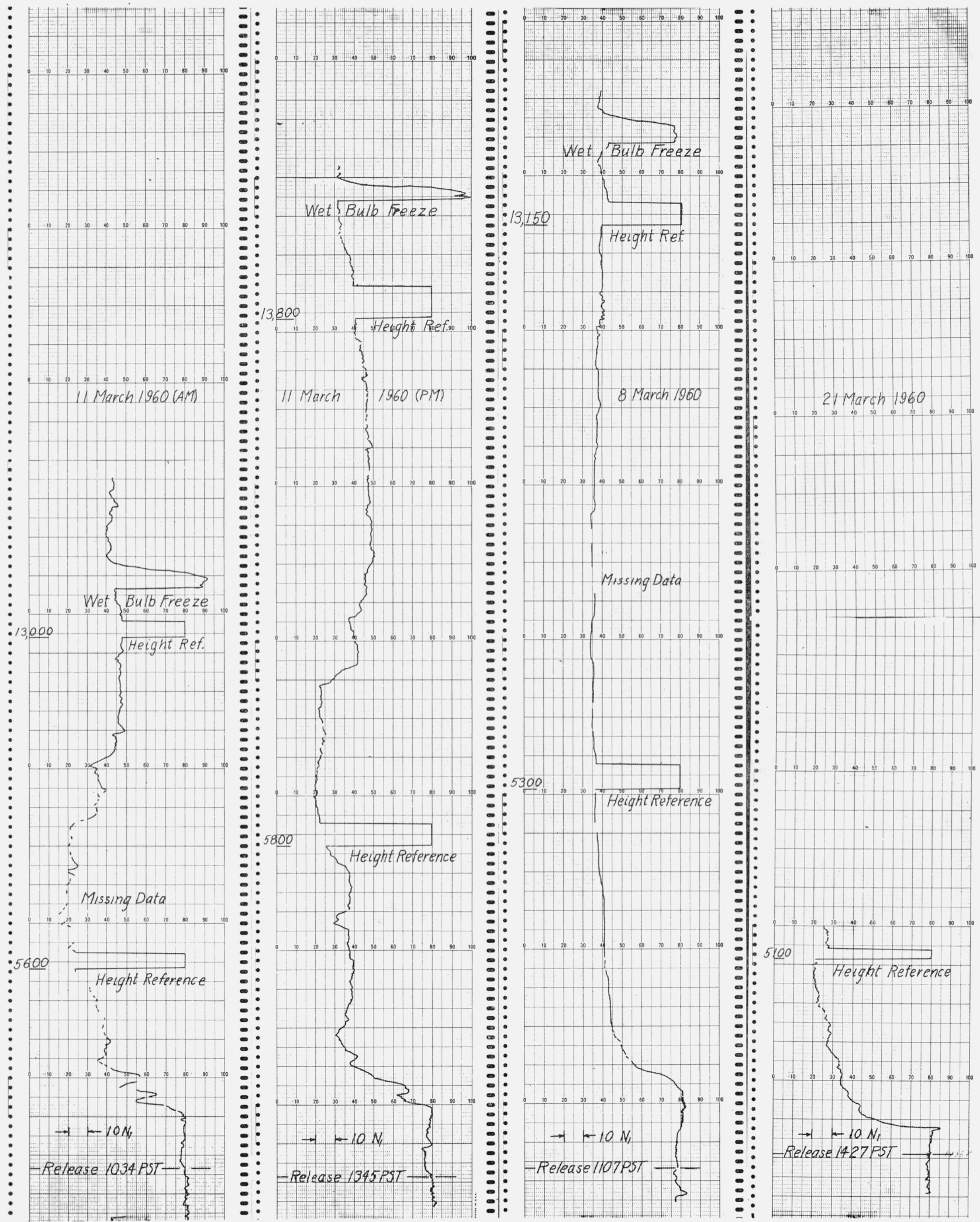

FiguRE 6. Sample records.

The missing data on the morning of 11 March occurred when the balloon came directly over the receivers and passed through the null of the receiving antennas. 


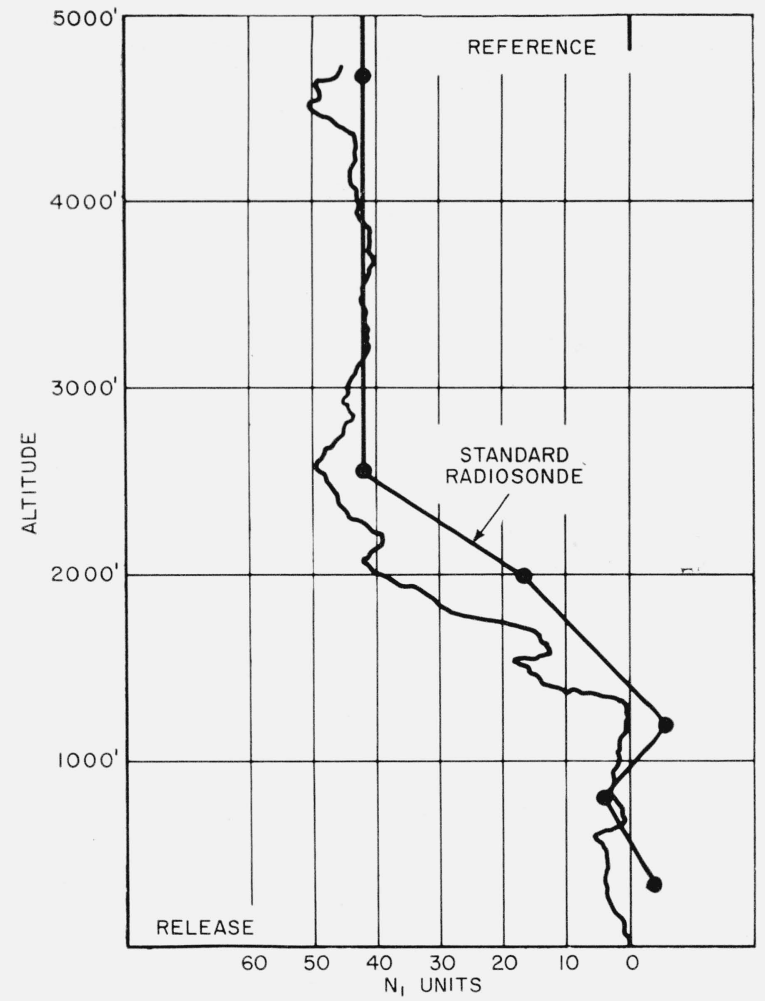

Figure 7. Afternoon sounding for 11 March 1960 and a standard radiosonde sounding taken immediately after the refractometer sounding (about one hour later).

The refractive index was computed by hand from the radiosonde data and is plotted in linear segments between computed points.

\section{Conclusions}

A meteorological refractometer system which is expendable and light enough to be flown on a radiosonde balloon can be built from standard radiosonde components. The system has proven to be very useful as a research tool for studying the microstructure of the atmosphere up to $13,000 \mathrm{ft} \mathrm{msl}$. Since the great majority of super refractive layers occur well below this height it should also prove to be a useful operational tool - especially in fleet units from which aircraft cannot be operated, and for problems in surface-to-air radar coverage where only the low troposphere is important.

\section{References}

[1] A. H. Clinger and A. W. Straiton, Adaptation of the radiosonde for direct measurement of radio refractive index, Bull. Am. Meteorol. Soc. 41, 250 (1960).

[2] J. Michealis and E. E. Gossard, Distribution of refractive layers over the North Pacific and Arctic, NEL Report 841 (1958).

[3] A. L. Crozier, Captive balloon refractovariometer, Rev. Sci. Instr. 29, 276 (1958)

[4] E. E. Gossard, Power spectra of temperature, humidity and refractive index from aircraft and tethered balloon measurements, IRE Trans. AP-8, 28 (1960).

[5] K. Hirao and K. Akita, A new type refractive index variometer, J. Radio Research Labs. (Japan) 4, (1957).

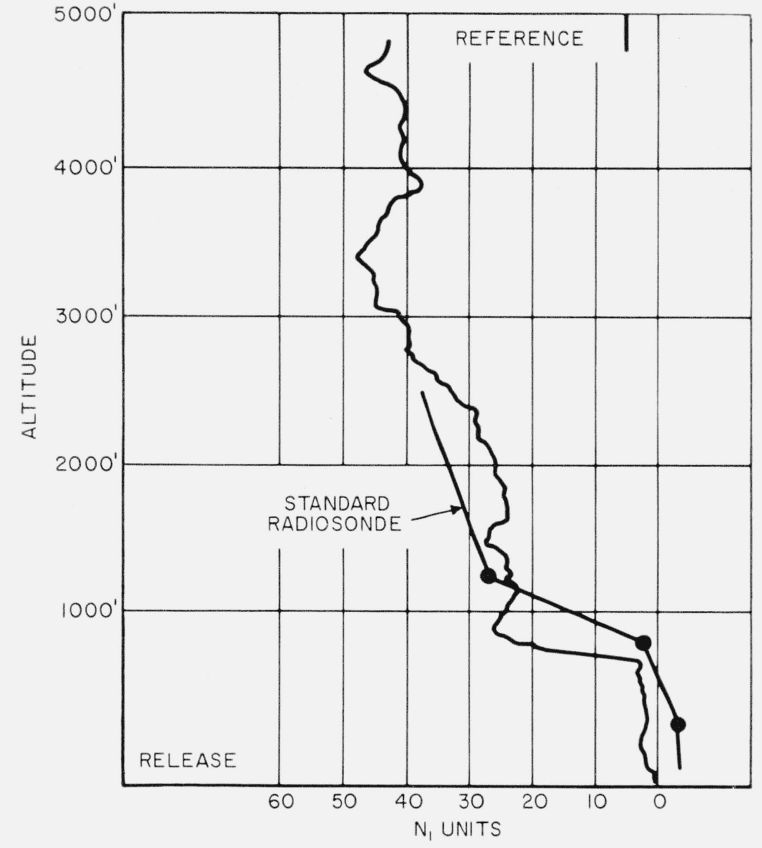

FiguRE 8. Refractometer sounding for 10 March 1960 and corresponding radiosonde data taken from the local San Diego raob.

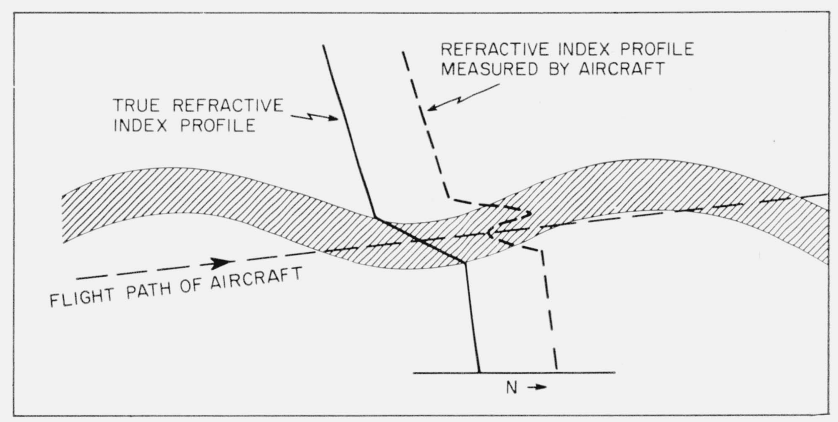

Figure 9. Schematic description of how an aircraft sounding can indicate an erroneous anomaly in the height distribution of refractive index. 